\title{
Reduced post-infarction myocardial apoptosis in women: a clue to their different clinical course?
}

\author{
G G L Biondi-Zoccai, A Abbate, R Bussani, D Camilot, F D Giorgio, M-P D Marino, F Silvestri, \\ F Baldi, L M Biasucci, A Baldi
}

$\mathrm{H}$ eart failure (HF) is less prevalent and has a better prognosis in women than in men. Also, the outlook after acute myocardial infarction (AMI) is more favourable in elderly women. ${ }^{1}$ Such sex related differences are probably related to sex specific cardiac remodelling processes. $^{2}$ However, despite several hypothetical pathogenetic mechanisms, there are no established explanations.

Myocardial apoptosis is a pivotal determinant of left ventricular (LV) dysfunction and cardiac failure in experimental and clinical studies, including after AMI. Sex may indeed influence apoptosis, as shown in normal aging and in end stage HF. ${ }^{3}$ However, little is known about the role of sex in post-infarction apoptosis. We thus evaluated the influence of sex on expression of pro-apoptotic mediators and myocardiocyte apoptotic index (AI) in subjects dying after AMI.

\section{METHODS}

Using established methods, ${ }^{4}$ we selected six females who had died after AMI with permanent infarct related artery occlusion at necropsy ( $<30$ hours after death); 15 males with similar characteristics were selected during the same period. Three men and one woman dying from non-cardiac causes were included as controls.

Re-infarction was excluded on clinicopathologic grounds in all cases. Infarct size was quantified at gross pathology on a four grade scale (small, moderate, moderate to extensive, and extensive).

Tissue specimens were obtained at peri-infarct and remote sites. In situ end labelling of DNA fragmentation by transferase-mediated biotinylated UTP nick end labelling (TUNEL) was performed (Apoptag, Oncor) and sections were stained with antibodies against muscle actin (DAKOCarpintera; dilution 1:50), and activated caspase-3 (Cell Signaling Technology; dilution 1:50). Myocardiocytes were defined as apoptotic by co-localisation of TUNEL and caspase-3. Immunohistochemical staining for proliferating cell nuclear antigen (PCNA) (DAKO; dilution 1:100) and SC-35 (Sigma; dilution 1:200) was also performed. AI was defined as the ratio of myocardiocytes co-expressing TUNEL and caspase- 3 positivity on nucleated cells per field $(\times 250)$, calculated on 100 random fields. Muscle actin negative cells, TUNEL positive but PCNA or SC-35 positive cells were not included in cell counts. Immunohistochemistry for myocardial expression of the proapoptotic mitochondrial protein bax was performed in 14 cases (four females, 10 males; Santa Cruz; dilution 1:100). Immunoreactivity was quantified as percentage of positive myocardiocytes per field $(\times 250)$ in an average of 50 fields. Immunohistochemical staining for the expression of cyclooxygenase-2 (COX-2), a potential marker of subacute myocardial ischaemia, was performed in four cases using a primary anti-COX-2 antibody (Santa Cruz; dilution 1:100). Results were described on a dicothomic (positive/negative) scale.

SPSS 10.1 was used as indicated for $\chi^{2}$ (Fisher corrected where appropriate), Mann-Whitney, Wilcoxon,
Kruskal-Wallis, and Spearman tests, with two tailed significance at 0.05 , were also used. Multivariable analysis was performed using a generalised linear model including univariate predictors of $\mathrm{AI}$ at the 0.10 level.

\section{RESULTS}

Demographic, clinical, and pathologic characteristics were not different between males and females (table 1).

Myocardial AI in peri-infarct regions correlated significantly with parameters of unfavourable LV remodelling (LV thickness, $R=-0.48, \mathrm{p}=0.029$, diameter to thickness ratio, $R=+0.56, \mathrm{p}=0.008)$. Comparing specific patterns of remodelling (from compensatory hypertrophy to eccentric hypertrophy to overt dilatation), AI appeared lower in subjects with favourable LV remodelling, and significantly higher in those with eccentric hypertrophy or overt dilation $(\mathrm{p}=0.046)$.

Myocardial AI in peri-infarct myocardium was 10-fold higher in men than in women, $25.9 \%(17.9-28.6) v 2.6 \%(0.5-$ 14.1), $\mathrm{p}=0.003$ (fig 1A). A non-significant trend towards a twofold increase in AI in men $(p=0.066)$ was found at remote sites (fig lB). Moreover, AI was significantly higher at peri-infarct versus remote regions in the overall population $(p<0.001)$, as well as in males $(p=0.001)$ and females only $(\mathrm{p}=0.027)$. Apoptotic myocardiocytes were extremely rare in control hearts $(0.01 \%, 0.01-0.02 \%)$, with no sex differences.

A higher number of myocardiocytes expressing cytoplasmic bax immunostaining was found in peri-infarct regions in males versus females, 55\% (44-61) v $14 \%$ (2.5-42), $\mathrm{p}=0.012$ ) (fig $1 \mathrm{C}$ ). A non-significant trend was also present in remote areas, $2.7 \%(0.8-4.1) \vee 0.9 \%(0.4-3.0)$, $\mathrm{p}=0.23$ ) (fig 1D). Moreover, bax expression at the periinfarct site was significantly associated with macroscopic signs of unfavourable LV remodelling such as thickness $(R=-0.68, \mathrm{p}=0.008)$, and diameter to thickness ratio $(R=+0.82, \mathrm{p}<0.001)$ (fig lE). The link between myocardial apoptosis, bax expression, and ischaemia was further strengthened by the finding of intense immunostaining for COX-2 myocardial expression in peri-infarct regions in subjects with higher than median myocardial apoptosis and bax staining (fig 2) (to see fig 2 visit the Heart websitehttp://www.heartjnl.com/supplement).

Multivariable analysis, assessing the independent role of the four borderline univariate predictors of increased periinfarct AI (age, sex, HF, and trauma as cause of death), showed maleness to be a significant predictor of AI $(\mathrm{p}=0.039)$.

Abbreviations: $\mathrm{AMI}$, acute myocardial infarction; $\mathrm{Al}$, apoptotic index; COX-2, cyclo-oxygenase-2; HF, heart failure; LV, left ventricular; PCNA, proliferating cell nuclear antigen; TUNEL, transferase mediated biotinylated UTP nick end labelling. 
Table 1 Clinical and pathologic characteristics

\begin{tabular}{lll}
\hline & $\begin{array}{l}\text { Females } \\
(\mathbf{n}=6)\end{array}$ & $\begin{array}{l}\text { Males } \\
(\mathbf{n}=15)\end{array}$ \\
\hline Clinical characteristics & $79(73-85)$ & $72(65-79)$ \\
$\quad$ Age (years) & $2(33 \%)$ & $9(60 \%)$ \\
Previous remote AMI (>6 months) (\%) & $15(12-24)$ & $25(12-50)$ \\
Time from AMI to death (days) & $4(66 \%)$ & $9(60 \%)$ \\
Fibrinolytic treatment (\%) & $2(33 \%)$ & $11(73 \%)$ \\
Symptomatic heart failure (\%) & 0 & $4(27 \%)$ \\
Trauma as cause of death (\%) & & $5(33 \%)$ \\
Pathologic characteristics & $3(50 \%)$ & $13(87 \%)$ \\
Anterior AMI (\%) & $6(100 \%)$ & $10(66 \%)$ \\
Transmural AMI (\%) & $6(100 \%)$ & 510 \\
*Large AMI (\%) & 500 & $(460-600)$ \\
Cardiac weight (g) & $(475-605)$ & 10.4 \\
Hamber diameter/wall & 8.6 & $(8.7-12.7)$ \\
thickness ratio & $(6.4-10.9)$ &
\end{tabular}

*Defined as an area of transmural necrosis involving more than one LV wall (approximately $30 \%$ of the circumference, or moderate to extensive, or extensive infarct) at pathology.

Data are expressed as median (interquartile range), unless otherwise stated; no significant differences were found between the two groups.

\section{DISCUSSION}

To the best of our knowledge, the present study shows for the first time that in subjects dying after AMI peri-infarct apoptosis is greater in men than women. In this clinicopathologic model, males also show increased post-infarction myocardial expression of bax in comparison to females.

HF is more frequent and severe in men than in women. ${ }^{1}$ Sex related differences in cardiac remodelling are probably among the explanations of such sex differences in clinical outcomes. $^{2}$ However, underlying mechanisms are still unclear, but may include the actions of oestrogens, telomerase, Akt, sodium/calcium exchanger, $\beta$ adrenergic receptors, and the renin-angiotensin system.

Similarly to findings by Guerra and colleagues, ${ }^{3}$ we show sex specific differences in AI after AMI. We can thus hypothesise that males and females may have a different modulation of the apoptotic pathway in the peri-infarct region; females could be partially protected from ischaemia induced bax expression and pro-apoptotic activity in comparison to men, similar to findings in cardiac fibroblasts. ${ }^{5}$

A cause-effect link between sex, apoptosis, and LV remodelling cannot however be demonstrated by the present observational data, and great caution should be applied when extrapolating our results to clinical practice as the study was limited by the small sample size and the non-experimental design.

In conclusion, myocardial apoptosis in peri-infarct areas is higher in males dying late after AMI than in females, and this difference is associated with increased myocardial bax expression. These findings may explain the more aggressive course of post-infarction HF in men and the relatively more
Infarct region

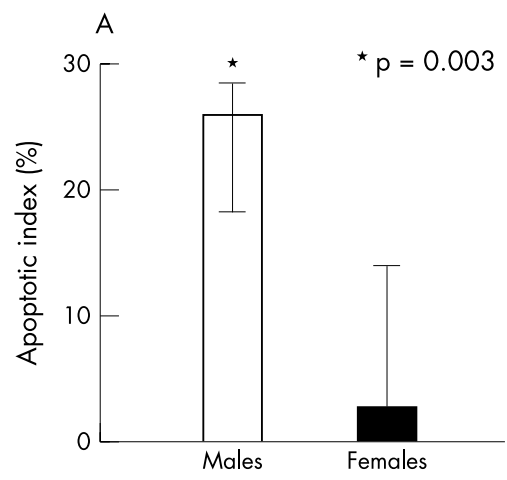

Remote region

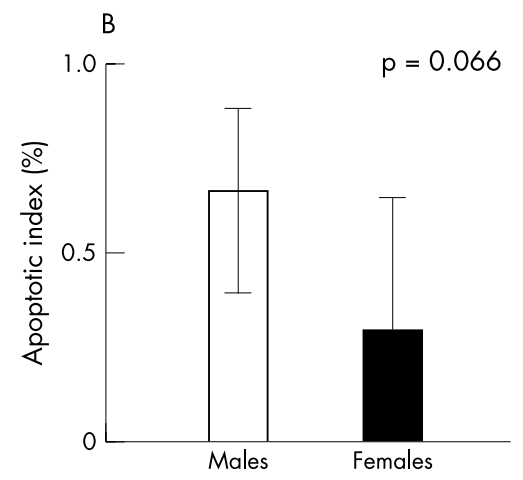

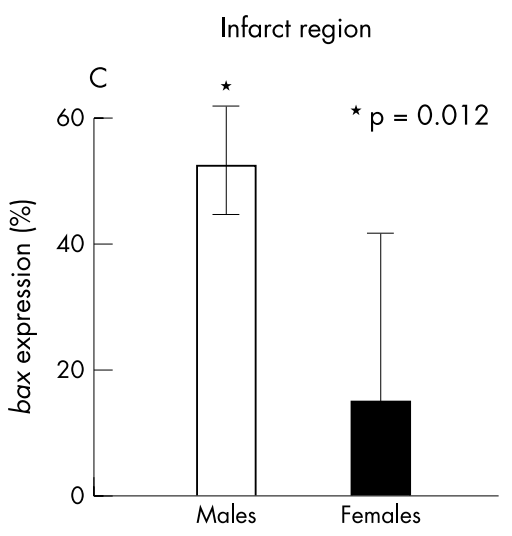
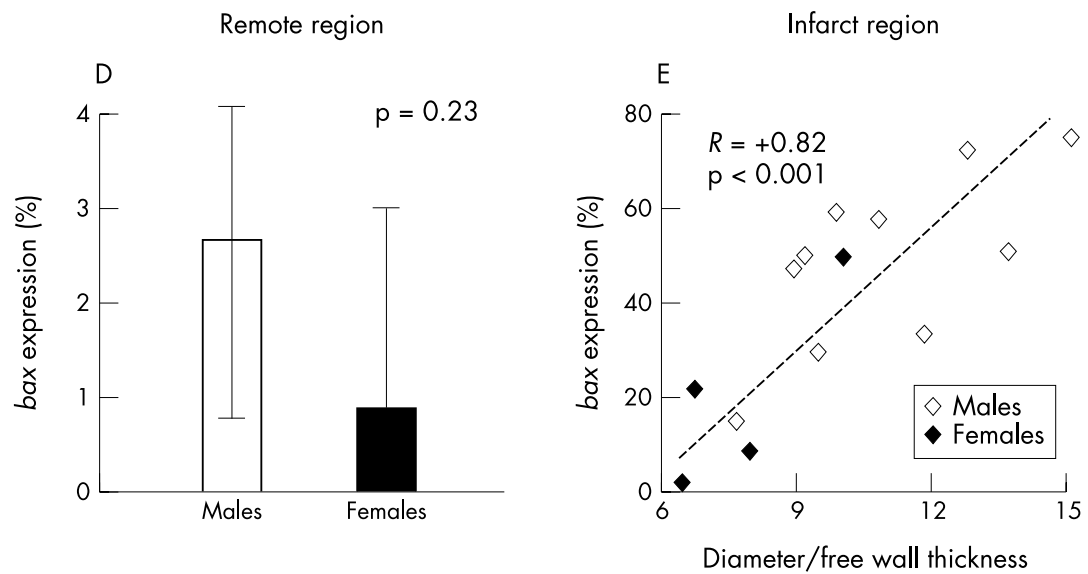

Figure 1 Sex and post-infarction apoptosis and bax expression in peri-infarct and remote myocardial region; (A) males (open columns) have a greater apoptotic index (Al) than females (solid columns) in peri-infarct region ( $p=0.003$ ); (B) men also have higher Al than women in remote myocardial region ( $p=0.066$ ); $(C)$ males ( $n=10$, open columns) have greater bax expression than females ( $n=4$, solid columns) in infarct regions $(p=0.012)$ and $(D)$ higher expression in remote myocardial regions $(p=0.23) ;(E)$ myocardial expression of bax in the infarct myocardium and signs of post-infarction LV remodelling expressed as transverse diameter to wall thickness ratio $(R=+0.82, \mathrm{p}<0.001)$. Males are shown as open diamonds, females as solid diamond. Column heights represent median values, whiskers represent interquartile ranges. 
benign post-infarction remodelling in women, potentially suggesting an increased resistance to ischaemia in females. The recognition and thorough characterisation of the influences of sex on cardiac disease may indeed provide keys to cardiovascular pathophysiology, which may eventually benefit both sexes.

\section{ACKNOWLEDGEMENTS}

This study was supported by MIUR, Second University of Naples and FUTURA Inc. grants.

Dr G Biondi-Zoccai presented this study as an abstract at the 2002 Italian Society of Cardiology Congress winning a Young Investigator Award.

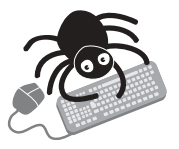

To view fig 2 please visit the Heart website-http:// www.heartinl.com/supplemental

\section{Authors' affiliations}

G G L Biondi-Zoccai, A Abate, L M Biasucci, Institute of Cardiology, Catholic University, Rome, Italy
R Bussani, D Camilot, F Silvestri, Department of Pathologic Anatomy, University of Trieste, Italy

F D Giorgio, Institute of Legal Medicine, Catholic University, Rome, Italy M-P D Marino, F Baldi, A Baldi, Department of Biochemistry and Biophysics, F Cedrangolo, Section of Pathologic Anatomy, Second University of Naples, Italy

Correspondence to: Dr Alfonso Baldi, Via G.Orsi, 25, 80128, Naples, Italy; alfonsobaldi@tiscali.it

Accepted 12 March 2004

\section{REFERENCES}

1 De Feo S, Opasich C. Comparison of the outcome in men and women with chronic heart failure. Ital Heart J 2003:4:511-3.

2 Olivetti G, Giordano G, Corradi D, et al. Gender differences and aging: effects on the human heart. J Am Coll Cardiol 1995;26:1068-79.

3 Guerra S, Leri A, Wang X, et al. Myocyte death in the failing human heart is gender dependent. Circ Res 1999;85:856-66.

4 Baldi A, Abbate A, Bussani R, et al. Apoptosis and post-infarction left ventricular remodeling. J Mol Cell Cardiol 2002;34:165-74.

5 Zhao X. Eghbali-Webb M. Gender-related differences in basal and hypoxiainduced activation of signal transduction pathways controlling cell cycle progression and apoptosis, in cardiac fibroblasts. Endocrine 2002; 18:137-45.

\section{IMAGES IN CARDIOLOGY}

doi: $10.1136 /$ hrt.2004.036194 Complete aortic dissection demonstrated by intraoperative transoesophageal echocardiography

A 75 year old man with a history of hypertension and coronary artery disease presented with syncope following a cardiac arrest at home. He was sent to our hospital from another centre with a diagnosis of aortic dissection. The patient was unconscious, intubated, and his overall condition was poor. He was sent to the operating room immediately where he suffered a severe hypotensive episode. Transoesophageal echocardiogram (TOE) revealed a linear structure going up and down from the aorta to the left ventricle. In the two chamber view at $90^{\circ}$ this structure appeared like a tube extending into the left ventricle (below left). In the left ventricular outflow track view at $120^{\circ}$ the complete dissection of the aorta from the aortic arch to the sinotubular junction was observed (below right). The dissection produced a severe aortic regurgitation with

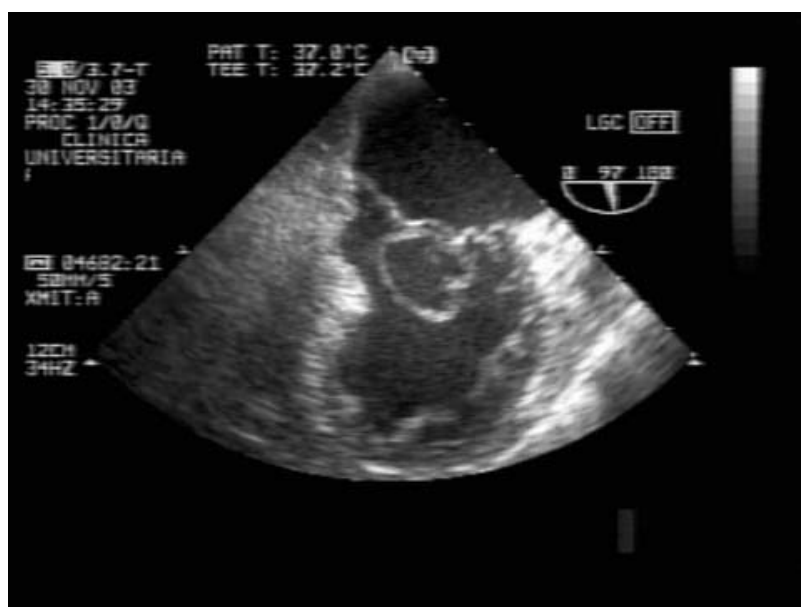

dilatation of the left ventricle. We performed a complete repair of the aortic arch with a supracoronary tube. The subsequent echocardiogram revealed only mild aortic regurgitation. However, the patient died five days later because of brain damage caused by the cardiac arrest.

A complete dissection of the aorta is not very common. In this case, the dissection was spectacular due to the complete separation of the intima, which produced these images on TOE.

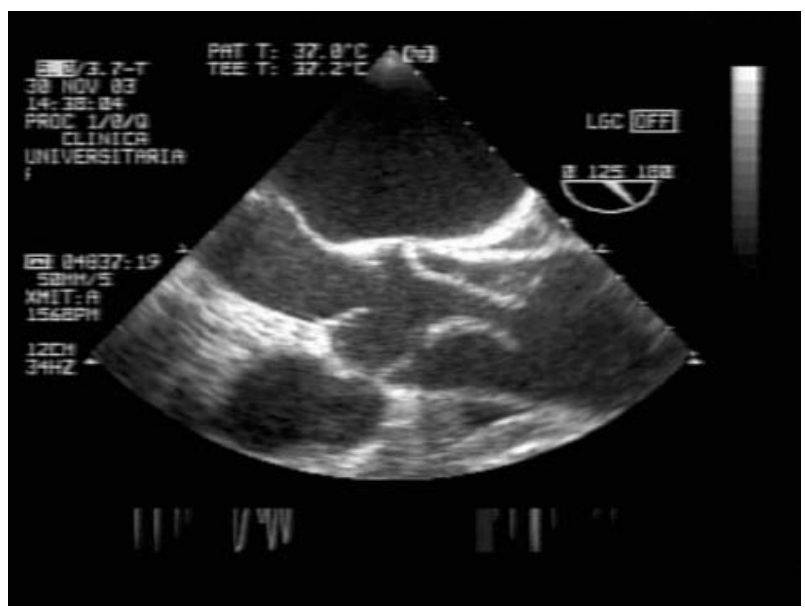

